Scientific Paper

\title{
Hybrid ANN optimized artificial fish swarm algorithm based classifier for classification of suspicious lesions in breast DCE-MRI
}

\author{
D JANAKI SATHYA ${ }^{1, a}$, K GEETHA $^{2}$ \\ ${ }^{1}$ Assistant Professor, Department of Electrical \& Electronics Engineering, PSG College of Technology, Coimbatore, India \\ ${ }^{2}$ Professor, Department of Electronics \& Communication Engineering, Karpagam College of Engineering, Coimbatore, India \\ ${ }^{a}$ E-mail address: janu_sathya@rediffmail.com
}

(received 29 may 2017; revised 4 September 2017; accepted 26 September 2017)

\begin{abstract}
Automatic mass or lesion classification systems are developed to aid in distinguishing between malignant and benign lesions present in the breast DCE-MR images, the systems need to improve both the sensitivity and specificity of DCEMR image interpretation in order to be successful for clinical use. A new classifier (a set of features together with a classification method) based on artificial neural networks trained using artificial fish swarm optimization (AFSO) algorithm is proposed in this paper. The basic idea behind the proposed classifier is to use AFSO algorithm for searching the best combination of synaptic weights for the neural network. An optimal set of features based on the statistical textural features is presented. The investigational outcomes of the proposed suspicious lesion classifier algorithm therefore confirm that the resulting classifier performs better than other such classifiers reported in the literature. Therefore this classifier demonstrates that the improvement in both the sensitivity and specificity are possible through automated image analysis.
\end{abstract}

Key words: Breast DCE-MR Images; lesion classification; classifier; Artificial Neural Networks; Artificial fishes swarm algorithm.

\section{Introduction}

Accurate segmentation and classification of lesion allows the physicians to identify suitable treatment. Cancer lesion classification methods have been explored over several decades [1]. The automatic classification between malignant and benign lesion is being explored in several different medical image analyses including: MRI, CT and X-ray mammography. The dynamic contrast enhanced (DCE)-MRI is an efficient imaging tool for analysis of the breast abnormalities. Computer-assisted evaluation (CAE) systems are beneficial tool for radiologists in identifying and evaluating lesions in DCE-MR images. These systems improve the reliability of clinical results. The CAE systems are designed by employing computer algorithms for automated lesion segmentation, trailed with proper edge enhancement technique such as unsharp filter, followed by proper thresholding [2]; the tumor or region of interest (ROI) is extracted efficiently from the edge enhanced image. As a result of segmentation, the region matching tumor or region of interest (ROI) is extracted from the corresponding breast DCEMR images. After segmentation of the ROI, optimal set of features are extracted from the ROI. The retrieved features are fed as input to the classifier to categorize whether the (ROI) lesion is benign or malignant.

Remaining part of this paper is structured as follows: Related works are discussed in section 2. Section 3 describes in detail the modules of the proposed suspicious lesion classification model. The result analyses are detailed in section 4. Conclusion of the proposed classifier is summed up in section 5.

\section{Related Works}

Up to this moment, lesion detection from breast DCE-MRI is still very challenging. The main reason is caused by that the lesions usually mix with the inhomogeneous tissues in the breast. The gray levels values of the inhomogeneous tissues in the breast might differ by way of presence of breast soft tissue. Hence, the gray level intensity of the surrounding tissues may be higher than those of the lesions in some cases, which leads to the miss detection if histogram measurement is used as the feature. However the density and gray levels values of the lesions are higher than the surrounding tissues, some lesion images contain blurred lesion edge and the contrast between the lesion boundaries and the other surrounding tissues are low. Furthermore, in few lesion images, the lesion might be enclosed by the surrounding tissues. This phenomenon increases the difficulty in the detection of lesions. For the lesion classifications, the main cause of difficulty is arisen by the diversification of features. In clinical diagnosis, the signs of abnormality observed by expert radiologists are very diverse. They include the size, contrast, intensity and density [1,3-15]. 
There are many classifiers available for classifying suspicious areas of breast DCE-MRI. Significant research on breast DCEMRI lesion classification methods have already been made to automatically predict lesions such as artificial neural networks, linear discriminant analysis, logistic regression and support vector machines [1,3]. The artificial neural networks based classifier have been one of the most popular approaches for investigating the classification of malignant and benign breast DCE-MR lesions [1,3-15]. The performance of any classifier depends on type of features used, training dataset provided and the type of classifier.

This paper presents a new classifier system constructed using artificial neural network optimized by AFSO algorithm to classify breast DCE-MRI lesion into benign or malignant using seven statistical textural features obtained from the lesion. The extracted statistical texture features were used to classify the lesion with a three-layered back propagation neural network. This work is the first experiment of artificial fish swarm optimization algorithm training the neural network for classifying the ROI (lesion) of breast DCE-MRI.

\section{Suspicious Lesion Classification Model}

The computer assisted lesion classification approach for breast DCE-MRI lesion detection typically comprised of two modules: (1) a quantitative feature extraction followed by feature selection and (2) a classifier that employs the features extracted from the lesion to discriminate lesion classes.

\subsection{Feature Extraction}

Once the ROI (region of interest) or tumor boundary is identified, characterization is necessary to determine the pathological nature of the lesion, i.e., whether the lesion is benign or malignant. For quantitative evaluation, many features have been retrieved from the lesions of DCE-MR images extracted during segmentation. A detailed procedure of segmentation phase has been reported in [16], shows the results of the segmentation, ROI selection procedure for mass or lesion extraction over the breast DCE-MR image with lesion.

The research work in quantitative morphological and kinetic features analysis of mass or lesions is considerably less, partly due to the difficulty in identifying relevant quantitative parameters that could characterize benign and malignant lesions. Texture feature has been proven to be useful in differentiating normal and abnormal pattern. For breast DCEMRI suspicious lesion classification statistical texture features are widely used [3,4,8-13,17-20].

In this proposed work, a set of 18 features were extracted from the ROI, 14 GLCM (gray level co-occurrence matrix) statistical texture measures as defined in [21] and 4 GLHM (gray level histogram moments) features defined in $[3,11]$. These features are used because they have proven improvement in classifier performance [3,11]. The features extracted are: entropy, skewness, difference moment, energy measure, difference variance, sum average, standard deviation, difference average, difference entropy, kurtosis, correlation, inertia, variance, inverse difference moment, mean, sum entropy, sum variance and information measure of correlation.

\subsection{Feature Selection}

Feature selection techniques are used for choosing a small feature set from available set of features, which will beneficial in reducing the size of the data to be processed and improving accuracy of the classifier. Selecting appropriate set of features for classification plays a most significant part in designing a good classifier. Hypothesis tests appear to be well suited for dealing with selection of most discriminative feature subset from the available feature set [3,11,20,22-27].

From the available 18 features, feature subset of 7 features is selected by hypothesis t-test; they are entropy, standard deviation, mean, skewness, kurtosis, variance and energy $[3,11]$. The features selected are listed with their mathematical equation for calculating its corresponding value from the extracted ROI.

\section{Entropy}

The Entropy, $\mathrm{H}$ is determined according to Equation 1.

$$
H=\frac{1}{M N} \sum_{i=1}^{M} \sum_{j=1}^{N} p(i, j)(-\ln p(i . j))
$$

Where $p(i, j)$ is the pixel value at point $(i, j)$ of an image.

\section{Standard Deviation}

The standard deviation, $\sigma$ is defined in Equation 2.

$\sigma=\sqrt{\frac{1}{M N} \sum_{i=1}^{M} \sum_{j=1}^{N}(p(i, j)-\mu)^{2}}$

\section{Mean Value}

The mean, $\mu$ is calculated using Equation 3.

$\mu=\frac{1}{M N} \sum_{i=1}^{M} \sum_{j=1}^{N} p(i, j)$

Where $p(i, j)$ is the pixel value at point $(i, j)$ of an image.

\section{Skewness}

The Skewness, S is calculated by Equation 4.

$$
S=\frac{1}{M N} \sum_{i=1}^{M} \sum_{j=1}^{N}\left[\frac{p(i, j)-\mu}{\sigma}\right]^{3}
$$

Where, $p(i, j)$ is the pixel value at point $(i, j), \mu$ and $s$ are the mean and standard deviation respectively.

\section{Kurtosis}

The Kurtosis, $\mathrm{K}$ is given in Equation 5.

$K=\left\{\frac{1}{M N} \sum_{i=1}^{M} \sum_{j=1}^{N}\left[\frac{p(i, j)-\mu}{\sigma}\right]^{4}\right\}-3$

Where, $p(i, j)$ is the pixel value at point $(i, j), \mu$ and $\sigma$ are the mean and standard deviation respectively. 


\section{Variance}

The Variance, $\mathrm{V}$ is calculated using the formula in Equation 6.

$V=\frac{1}{M N} \sum_{i=1}^{M} \sum_{j=1}^{N}(p(i, j)-\mu)^{2}$

Where $p(i, j)$ is the pixel value at point $(i, j)$ of an image. $\mu$ is the mean.

\section{Energy}

The Energy, E is calculated using Equation 7.

$$
E=\frac{1}{M N} \sum_{i=1}^{M} \sum_{j=1}^{N} p^{2}(i, j)
$$

Where $p(i, j)$ is the pixel value at point $(i, j)$ of an image.

The seven statistical texture features extracted from lesion region are fed as input to the classifier module of the classification system for efficient discrimination between the lesion types.

\subsection{Proposed Lesion Classifier}

The classifier used in designing lesion classification module is based on artificial neural network and the artificial fish swarm algorithm is used to optimize the neural network. The classifier chosen is a three layered backpropagation neural network.

\section{Artificial Neural Network Architecture}

The architecture artificial neural network based classifier comprises of a three-layer back propagation neural network is shown in Figure 1. The three-layer back propagation neural network comprise of an input layer, an output layer and a hidden layer. The number of neurons in the input layer depends to the number of input features, since the input feature set consist of seven features, the input layer has 7 neurons. The output layer consist one neuron whose values varies between zero or one which specifies the level of malignancy, where zero means benign and one means malignant. The number of neurons in hidden layer is identified by trial-and-error runs and the neural network architectures with five hidden neurons are selected.

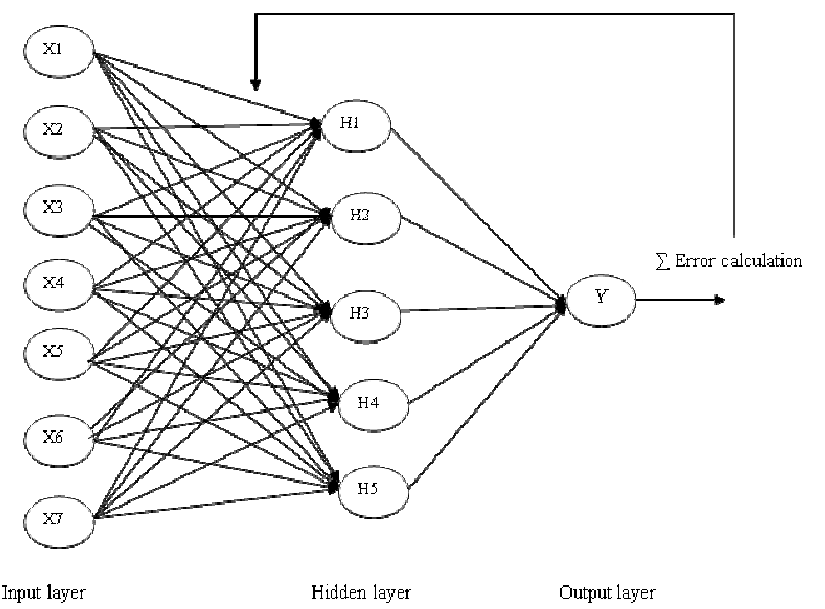

Figure 1. Architecture of the proposed Artificial Neural Network based Lesion classifier.
The learning coefficient of 0.0001 is set for the hidden layer and 0.001 for the output layer. The linear and hyperbolic tangent transfer functions are used as activation functions of hidden and output layers of neural network respectively. The parameters are estimated through trial and error evaluations. Therefore, the ANN structure with the lowest error value is considered as an optimal structure.

The error backpropagation algorithm is the common method for training neural network when used as classifier. During the training phase, the feed forward computation is done and compared with the desired output; the error obtained is backward propagated to adjust the weights. For training the neural network the fish swarm optimization algorithm is used to optimize the weights of the neural network in place of the backpropagation procedure.

Basically, neural network training is a process by which the synaptic weights $\mathrm{W}$ of an ANN are adjusted in an incessant procedure of stimulation by the background in which the network is implanted. The type of training or learning procedure decides the method in by which the synaptic weight change takes place. This proposed classifier model emphasis on supervised learning procedure, which utilizes a labeled set of training database made up of $\mathrm{X}$ input-output samples.

The back propagation neural network training process is to reduce the error value. The error value is the difference between the actual attained output value and the desired output value of the neural network for the given set of training input patterns. Training of the neural network is carried out by providing to the network a set of training pattern of known desired output are taken from the training database. The error value for each training pattern is the sum of the squared differences between the desired and actual attained outputs of the network corresponding to the provided input training pattern. The above process is continued for all the patterns in the training database and the error values for all the training patterns are added to achieve the error value for a back propagation neural network.

\section{Artificial Fish Swarm Optimization Algorithm}

Artificial Fish Swarm Optimization (AFSO) is an optimization algorithm, it was originally designed and implemented in 2002 [28]. The AFSO algorithm designed based on imitating the behaviors of fish swarm. It also relies on a population based search methodology, which consists of a number of fishes. Each individual fish explores the problems solution space to find an optimum solution and is referred to as an artificial fish (AF). AFSO algorithm solves a problem based on four major process of fish swarm, they are preying, swarming, following, and random, these four processes performed by AF individuals to achieve a global optimum solution without loss of generality. AF individual will freely move in the direction of possible position within its visual limit and each movement depends on the Step-limit; Step and Visual parameters generate great impact on AFSO algorithm performance. The position, in which AF exist is considerably the solution, If the solution 
obtained is better than already existing one then the new solution is memorized, else the old value is retained. The consistency of the solution is determined by calculating the fitness of the solution. AFs attain a solution point where its amount of food consistency is maximum, which is also, called as global optimum solution. [28]. AFSO algorithm has been used for solving several kinds of optimization problems; it has also shown to provide better performance [28-35].

\section{Neural Network Training using the Fish Swarm Optimization Algorithm}

The training of neural network using the artificial fish swarm algorithm will comprise of the swarm of fish searching for the best values of the synaptic weights assigned between the neurons within the neural network; each fish represents a neuron in the network with a particular set of weight vectors. The purpose of the algorithm is to find the fish swarm producing optimal weight vectors, which in turn provides the smallest error value. The neural network error value $E$ is defined by Equation 8:

$E(w(i))=\frac{1}{n} \sum_{j=1}^{n} \sum_{m=1}^{m}\left(D_{m}-A_{m}\right)$

Where $\mathrm{E}(\mathrm{w}(\mathrm{i}))$ is the error value at the $\mathrm{i}^{\text {th }}$ iteration; w(i) is the weights in the network connections at the $i^{\text {th }}$ iteration; $D_{m}$ is the desired output value of the $m^{\text {th }}$ neuron of the network; $A_{m}$ is the actual value of the $m^{\text {th }}$ output neuron of the network; $m$ is the number of output neurons; and $\mathrm{n}$ is the number of training patterns.

The artificial neural network training using the fish swarm optimization algorithm consists of the following steps:

step1: Initialization: Generate $\mathrm{N}$ individuals randomly. The fish population size of $\mathrm{N}$ is defined as $\mathrm{x}=\left(\mathrm{x}_{1}, \mathrm{x}_{2}, \ldots, \mathrm{x}_{\mathrm{n}}\right)$.

step2: Fitness value calculation: calculate the corresponding fitness value to every individual fish using Equation 9; select the fittest solution of the artificial fish.

$$
f_{1}(x)=\sum_{i=1}^{n} x_{i}^{2}
$$

\section{step3: Repeat from step 4 to step 7.}

step4: Apply the training dataset to the neural network to determine the error value. This achieved by following steps:

(i) Produce new solutions and compare the fitness of the new solutions and select the corresponding behavior then optimal solution is added in the next generation Gen=Gen+l using Equations 10 and 11.

$$
X_{i}^{v}=X+\text { Visual.rand }()
$$$$
X_{N E X T}=X+\frac{X_{V}-X}{\left\|X_{V-X}\right\|} \text {.Step.rand () }
$$

(ii) Calculate the error value between the target and obtained value. step5: Based on the error value obtained from step 4,

(i) update the AFs location to the new value if it is better than the previous value; else, the location of AFs is kept unchanged.

(ii) Remember the best AFs location achieved so far.

step6: Stop the process of weight updation, if the error value has dropped below a predetermined threshold or after finishing the defined number of iterations.

step7: Else, return to step 4.

step8: Stop.

In the proposed artificial neural network training using fish swarm optimization algorithm, each cycle of the search process for optimized weights consists of three steps after population and control parameter initialization for ANN-AFSO algorithm. The artificial fish swarm would continue the searching process until the last cycle to find the optimal weights for artificial neural networks. The AFs location of which has low food concentration is neglected is replaced with a new AFs location. Every artificial fish would produce new solutions (weights) for the network.

The AFs location is applied randomly and is initialized for evaluation, which uses sphere function given in Equation 9 to evaluate the fitness. Every fish try produce new evaluated AFs location for the network and the fitness evaluation is used to select the best food source location. If the new AFs location has equal or better fitness than the old AFs location, it is replaced with the new AFs location in the memory. Otherwise, the old AFs location is retained in the memory. The proposed structure can be effectively used for breast DCE-MRI lesion classification task.

\section{Experimental Results and Analysis}

This section presents the visual and statistical experimental results and analysis of the proposed classifier for classifying the suspicious lesion on real breast DCE-MR Image dataset.

\subsection{Evaluation Data and Methods}

The breast DCE-MR image dataset contains records of 120 patients. The provide image dataset includes 80 malignant lesions and 40 benign lesions. The breast DCE-MRI dataset is received from the Radiology Department of Kovai Medical Center and Hospital (KMCH), Coimbatore, India. The performance of the proposed classifier model is validated using cross-validation experimentation and Leave-one-out- cross-validation, for cross-validation the image dataset is divided into the training set and the test set. The training sets of 70 images from the dataset (with 50 malignant lesions and 20 benign lesions) were used to construct the classifier model and the test sets of 50 images from the dataset (with 30 malignant lesion and 20 benign lesions) are used to evaluate the trained classifier model. The dataset images used for training are not used to test the classifier model. For leave-one out-crossvalidation experimentation, if $n$ images are there in dataset, $n-1$ images in the dataset are used for training the classifier and the 
left out image is used for testing the classifier, this process is repeated $\mathrm{n}$ times, where each time a different image is left out. This method of experimentation takes more time.

\subsection{Performance Evaluation}

The classification performance can be assessed in terms of the sensitivity, specificity, accuracy and Error in classification of the system as derived from the Equations 12-15.

Accuracy: $A C C=\frac{T P+T N}{T P+F P+F N+T N}$

Specificity (SP): $S P=\frac{T N}{T N+F P}$

Sensitivity $(\mathrm{SN}): S N=\frac{T P}{T P+F N}$

Error in classification: $E=1-$ Accuracy

Eq. 15

Where $\mathrm{TP}=$ true positive, $\mathrm{TN}=$ true negative, $\mathrm{FP}=$ false positive, and $\mathrm{FN}=$ false negative. It is clear that the main purpose of any classifiers to reduce the false positive and negative rates, similarly, to exploit maximum true negative and positive rates.

The results obtained by the proposed fish swarm algorithm trained neural network classifier using both validation methods are illustrated in Table $\mathbf{1}$ and Table 2 . There exist variation in performance results between the leave-one-out cross-validation method and the cross-validation experimentation is due shrinkage of the training set use in cross-validation method. The results of both validation methods indicate that the selected texture features for classifier provides a better discriminating between benign and malignant lesions. Table 3 presents the parameter values used in the proposed artificial fish swarm optimized training algorithm implementation. The parameter values were decided empirically.

The sensitivity of the algorithm is high since the rate of accuracy is higher. The efficiency of the proposed algorithm is good, because of less number of parameters used and less space required. The proposed algorithm represented the advantages of achieving global optimum solution and high accuracy.

The seven statistical texture features values are computed over the image containing malignant and benign lesions and are tabulated in Table 4. This preliminary result shows that the texture features values of the malignant lesions and the benign lesions are different. This is also indicates that the selected texture features are more effective in the lesion classification for the given image database.

\subsection{ROC Analysis of the Classifier Performance}

The accuracy of any classifier model is generally assessed by using ROC analysis [36]. The ROC analysis curve is obtained by plotting of the sensitivity values versus the 1-specificity values of the classifier result at differing thresholds. The accuracy of any classifier is measured by area under the ROC curve (AUC) termed AZ. The estimation of the AZ value can be obtained with the trapezoidal rule which can underestimate the areas under the ROC curve.

Table 1. Detailed investigation of the benign vs. malignant classification of the proposed lesion classifier algorithm.

\begin{tabular}{ccc}
\hline \hline Evaluation type & $\begin{array}{c}\text { Cross- } \\
\text { validation }\end{array}$ & $\begin{array}{c}\text { Leave-one-out- } \\
\text { cross-validation }\end{array}$ \\
\hline Number of cases used for classification & 50 & 120 \\
True positive & 29 & 79 \\
True negative & 18 & 39 \\
False positive & 2 & 1 \\
False negative & 1 & 1 \\
Sensitivity (\%) & 96.99 & 98.75 \\
Specificity (\%) & 90 & 97.5 \\
Accuracy (\%) & 94 & 98.33 \\
Error in classification (\%) & 6 & 1.667 \\
No of cases misdiagnosed & 3 & 2 \\
\hline \hline
\end{tabular}

Table 2. Simulation outcomes of lesion classification algorithm.

\begin{tabular}{ccccc}
\hline \hline \multirow{2}{*}{ Type of Experimentation } & \multicolumn{2}{c}{$\begin{array}{c}\text { Correct classification } \\
(\boldsymbol{\%})\end{array}$} & $\begin{array}{c}\text { Misclassification } \\
(\boldsymbol{\%})\end{array}$ \\
\cline { 2 - 5 } & Benign & Malignant & Benign Malignant \\
\hline Cross-validation & 90 & 96.99 & 10 & 3.01 \\
Leave-one-out- cross-validation & 97.5 & 98.75 & 2.5 & 1.25 \\
\hline \hline
\end{tabular}

Table 3. Parameters used in ANN Optimized AFSO training algorithm.

\begin{tabular}{cc}
\hline \hline Parameters & Values \\
\hline Fish swarm population size & 200 \\
Visual range of artificial fish & 40 \\
Crowding factor & 0.95 \\
Step factor each fish in fish swarm & 8 \\
Genmax (maximum generation) & 35 \\
Maximum number of iterations, R & 2000 \\
\hline \hline
\end{tabular}

Table 4. Statistical texture features computed from the image dataset (malignant and benign lesions).

\begin{tabular}{|c|c|c|c|c|c|c|c|}
\hline \multirow[b]{2}{*}{ Image } & \multicolumn{7}{|c|}{ Features } \\
\hline & Entropy & $\begin{array}{l}\text { Standard } \\
\text { Deviation }\end{array}$ & Mean & Skewness & Kurtosis & Variance & Energy \\
\hline Malignant lesion & 0.0208 & 8.9189 & 180.50 & 18.209 & 43.439 & 490.66 & 103015 \\
\hline Benign lesion & 0.079 & 16.791 & 148.53 & 7.1439 & 65.1719 & 339.69 & 149259 \\
\hline
\end{tabular}


The ROC curve has been plotted by varying the threshold values of the output layer of the classifier is shown in Figure 2. The threshold value is varied from 0.34 to 0.74 in steps of 0.02 . The proposed classifier achieves $\mathrm{A}_{\mathrm{Z}}=0.989$ using leave-oneout cross-validation experimentation approach and $\mathrm{A}_{\mathrm{Z}}=0.973$ using cross-validation experimentation, which illustrates that the obtained classification performance by both validation methods are optimum.

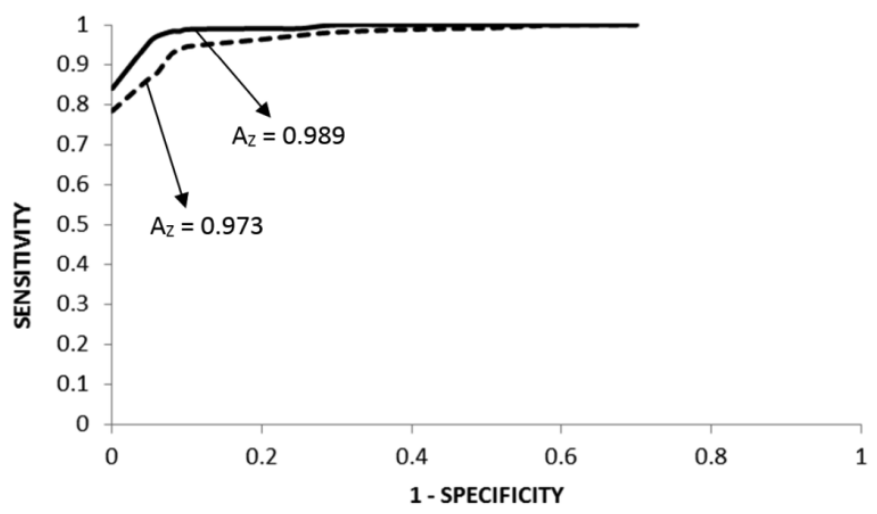

Figure 2.The receiver operating characteristic (ROC) curves of the proposed ANN optimized artificial fish Swarm algorithm based classifier for two validation methods. The dotted line denotes the ROC curve obtained using cross-validation method with $\mathrm{AZ}$ of 0.973 . The solid line denotes the ROC curve obtained using leave-one-out cross-validation method with $\mathrm{AZ}$ of 0.989 .

\subsection{Comparing Performance of Different Classifiers}

The accessibility of numerous high-quality classifier models would embolden the physicians to implement these computer assisted tools into every day medical examination. Classifiers performance has been compared in order to determine the best classifier for discriminating between lesion types. Table 5 lists the highest performing classifiers using texture features [8,10,18 and 19] for breast lesion classification in DCE-MRI. The performance is measured in terms of accuracy, sensitivity and specificity. The table also lists the type of validation used to evaluate the classifier, type of classifier used in each case and the number of malignant and benign data samples used. It should be noted that the breast DCE-MR image datasets used by techniques in Table 5 are different and results only illustrates the qualitative perception.

By investigating the performance results of the proposed classifier, the best results are achieved when using ANN trained artificial fish swarm optimization algorithm based classifier. AFSO trained ANN classifier outperforms all other existing classifiers. The results of the experiments also suggest that combining different types of information (GLCM and GLHM) into single feature set may enhance the discriminatory power of a feature space without increasing its size. This will lead to an automated computer assisted classification system capable of discriminating lesion in breast DCE-MRI.

Table 5. Qualitative performance comparison of the highest performing classifiers using statistical texture features reported in the literature.

\begin{tabular}{|c|c|c|c|c|}
\hline Classifier type & Dataset size & Accuracy (\%) & Sensitivity (\%) & Specificity (\%) \\
\hline $\begin{array}{l}\text { Artificial neural network (ANN) } \\
\text { (Leave-one-out- cross -validation) }\end{array}$ & $\begin{array}{c}\text { Dataset }=112 \\
26-\text { Benign } \\
86-\text { Malignant }\end{array}$ & 80 & 82 & 72 \\
\hline $\begin{array}{c}\text { Logistic regression (LR) } \\
\text { (Leave-one-out- cross - validation) }\end{array}$ & $\begin{array}{l}\text { Dataset }=104 \\
\text { 35- Benign } \\
\text { 69- Malignant }\end{array}$ & 80 & 91.2 & 64.5 \\
\hline $\begin{array}{l}\text { Support vector machine (SVM) } \\
\text { (Leave-one-out- cross -validation) }\end{array}$ & $\begin{array}{c}\text { Dataset }=112 \\
26-\text { Benign } \\
86-\text { Malignant }\end{array}$ & 81 & 90 & 75 \\
\hline $\begin{array}{l}\text { Linear discriminant analysis (LDA) } \\
\text { (Leave-one-out- cross -validation) }\end{array}$ & $\begin{array}{c}\text { Dataset }=121 \\
44-\text { Benign } \\
77-\text { Malignant }\end{array}$ & 88 & 90 & 79 \\
\hline $\begin{array}{l}\text { Artificial Bee colony algorithm optimized ANN classifier } \\
\text { (Cross-validation) }\end{array}$ & $\begin{array}{c}\text { Dataset }=85 \\
20-\text { Benign } \\
\text { 65- Malignant }\end{array}$ & 91.42 & 92.30 & 88.88 \\
\hline
\end{tabular}




\subsection{Snap Shots of CAE system for Breast DCE-MR Images}

This section shows few snap shots of graphical user interface of the classification system explained.
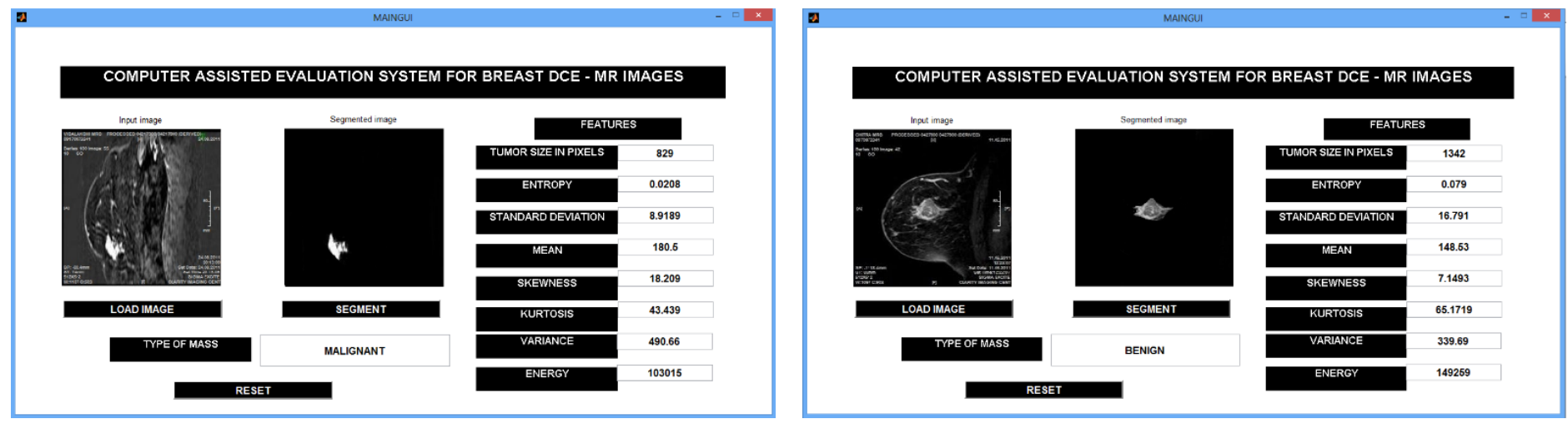

Figure 3. Graphical user interface of the proposed classification system.

\section{Conclusion}

This paper has proposed the best performing features and classifiers for classifying benign and malignant lesions in DCE-MRI of the breast. A set of 18 textural features is proposed. These are based on statistical textural features mainly GLCM (gray level co-occurrence matrix) and GLHM (gray level histogram moments) information from the DCEMRI data. The features subset is selected using statistical hypothesis t-test, which results in indicating seven features are enough to best in discriminating between benign and malignant. The designed AFSO optimized ANN classifier yields an (AUC) $\mathrm{A}_{\mathrm{Z}}=0.989$ using leave-one-out crossvalidation experimentation approach and $\mathrm{A}_{\mathrm{Z}}=0.973$ using cross-validation experimentation approach on the given validation dataset, which declares that the proposed classifier is actually a beneficial tool for the diagnosis of the breast cancer than several existing classifiers.

\section{Acknowledgement}

We thank Dr R. Rupa, DMRD, DNB, Consultant Radiologist, $\mathrm{KMCH}$ (Kovai Medical Centre and Hospital), Coimbatore, Tamil Nadu, India, for providing the breast DCE-MR images dataset along with its ground truth results used for testing the classifier algorithm proposed.

\section{References}

[1] Fusco R, Sansone M, Petrillo A, Sansone C. A multiple classifier system for classification of breast lesions using dynamic and morphological features in DCE-MRI. Statistical Techniques in Pattern Recognition (SPR) and Structural and Syntactic Pattern Recognition (SSPR) 2012 Nov 7 (pp. 684-692). Springer Berlin Heidelberg.

[2] Weszka JS. A survey of threshold selection techniques. Computer Graphics and Image Processing. 1978;7(2):259-265.

[3] Sathya J, Geetha K. Experimental investigation of classification algorithms for predicting lesion type on breast DCE-MR images. Int J Comp App. 2013;82(4):1-8.

[4] Gibbs P, Turnbull LW. Textural analysis of contrast-enhanced MR images of the breast. Magn Reson Med. 2003;50(1):92-98.

[5] Degenhard A, Tanner C, Hayes C, et al. Comparison between radiological and artificial neural network diagnosis in clinical screening. Physiol Meas. 2002;23(4):727-739.

[6] Iftikhar K, Anwar S, Khan MT, Akbar SR. An Optimal Neural Network Based Classification Technique for Breast Cancer Detection. Journal of Engineering and Applied Sciences (JEAS), University of Engineering and Technology, Peshawar. 2016;35(1):51-58.

[7] Tzacheva AA, Najarian K, Brockway JP. Breast cancer detection in gadolinium-enhanced MR images by static region descriptors and neural networks. J Magn Reson Imaging. 2003;17(3):337-342.

[8] Meinel LA, Stolpen AH, Berbaum KS, et al. Breast MRI lesion classification: Improved performance of human readers with a backpropagation neural network computer-aided diagnosis (CAD) system. J Magn Reson Imaging. 2007;25(1):89-95.

[9] Keyvanfard F, Shoorehdeli MA, Teshnehlab M. Feature selection and classification of breast cancer on dynamic magnetic resonance imaging using ANN and SVM. American Journal of Biomedical Engineering. 2011;1(1):20-25.

[10] Juntu J, Sijbers J, De Backer S, et al. Machine learning study of several classifiers trained with texture analysis features to differentiate benign from malignant soft-tissue tumors in T1-MRI images. J Magn Reson Imaging. 2010;31(3):680-689.

[11] Sathya J, Geetha K. Mass classification in breast DCE-MR images using an artificial neural network trained via a bee colony optimization algorithm. ScienceAsia. 2013;39(3):294-305. 
[12] Sawant HK, Shinde VD. Breast tumor analysis using texture features and wavelet transform with dynamic neural network based training. J Inf Knowl Res Comput Eng. 2011;1(2):46-51.

[13] Agner SC, Soman S, Libfeld E, et al. Textural kinetics: a novel dynamic contrast-enhanced (DCE)-MRI feature for breast lesion classification. J Digit Imaging. 2011;24(3):446-463.

[14] Vomweg TW, Buscema M, Kauczor HU, et al. Improved artificial neural networks in prediction of malignancy of lesions in contrast-enhanced MR-mammography. Med Phys. 2003;30(9):2350-2359.

[15] Bhooshan N, Giger ML, Jansen SA, et al. Cancerous breast lesions on dynamic contrast-enhanced MR images: computerized characterization for image-based prognostic markers. Radiology. 2010;254(3):680-690.

[16] Sathya DJ, Geetha K. Development of Intelligent System Based on Artificial Swarm Bee Colony Clustering Algorithm for Efficient Mass Extraction from Breast DCE-MR Images. Int J on Recent Trends in Engineering and Technology. 2011;6(1):82-88.

[17] Woods BJ, Clymer BD, Kurc T, et al. Malignant-lesion segmentation using 4D co-occurrence texture analysis applied to dynamic contrast-enhanced magnetic resonance breast image data. J Magn Reson Imaging. 2007;25(3):495-501.

[18] Gal Y, Mehnert A, Bradley A, et al. Feature and classifier selection for automatic classification of lesions in dynamic contrastenhanced MRI of the breast. In: Digital Image Computing: Techniques and Applications, 2009. DICTA'09. 2009 Dec 1 (pp. $132-$ 139). IEEE.

[19] Yao J, Chen J, Chow C. Breast tumor analysis in dynamic contrast enhanced MRI using texture features and wavelet transform. IEEE Journal of Selected Topics in Signal Processing. 2009;3(1):94-100.

[20] Loose J, Harz MT, Laue H, et al. Assessment of texture analysis on DCE-MRI data for the differentiation of breast tumor lesions. In: SPIE Medical Imaging 2009 Feb 26 (pp. 72600K-72600K). International Society for Optics and Photonics.

[21] Haralick RM, Shanmugam K. Textural features for image classification. IEEE Transactions on Systems, Man, and Cybernetics. 1973;3(6):610-621.

[22] Vickers AJ. Parametric versus non-parametric statistics in the analysis of randomized trials with non-normally distributed data. BMC Med Res Methodol. 2005;35(5):1-12.

[23] Haury AC, Gestraud P, Vert JP. The influence of feature selection methods on accuracy, stability and interpretability of molecular signatures. PloS One. 2011;6(12):e28210.

[24] Foody GM. Classification accuracy comparison: hypothesis tests and the use of confidence intervals in evaluations of difference, equivalence and non-inferiority. Remote Sensing of Environment. 2009;113(8):1658-1663.

[25] Liu H, Li J, Wong L. A comparative study on feature selection and classification methods using gene expression profiles and proteomic patterns. Genome Inform. 2002;13:51-60.

[26] Satake H, Nishio A, Ikeda M, et al. Predictive value for malignancy of suspicious breast masses of BI-RADS categories 4 and 5 using ultrasound elastography and MR diffusion-weighted imaging. AJR Am J Roentgenol. 2011;196(1):202-209.

[27] Eker OF, Camci F, Kumar U. Failure diagnostics on railway turnout systems using support vector machines. In: International Workshop and Congress on eMaintenance: 22/06/2010-24/06/2010 2010 (pp. 248-251). Luleå tekniska universitet.

[28] Li XL, Shao ZJ, Qian JX. An optimizing method based on autonomous animats: fish-swarm algorithm. System Engineering Theory and Practice. 2002;22(11):32-38.

[29] de Oliveira J, Ludermir T. A modified artificial fish swarm algorithm for the optimization of extreme learning machines. Artificial Neural Networks and Machine Learning-ICANN 2012. 2012:66-73.

[30] Chen H, Wang S, Li J, Li Y. A hybrid of artificial fish swarm algorithm and particle swarm optimization for feed forward neural network training. Proceedings of International Conference on Intelligent Systems and Knowledge Engineering 2007. Advances in Intelligent Systems Research, Atlantic Press. 2007.

[31] Yang Wang, Wei Zhang, Hongxing Li. Application of artificial fish swarm algorithm in image registration. Computer Modelling \& New Technologies. 2014;18(12B):510-516.

[32] Hasan S, Quo TS, Shamsuddin SM, Sallehuddin R. Artificial Neural Network Learning Enhancement Using Artificial Fish Swarm Algorithm. In: Proceedings of the 3rd International Conference on computing and Informatics, ICOCI 2011 (pp. 8-9).

[33] Wang CR, Zhou CL, Ma JW. An improved artificial fish-swarm algorithm and its application in feed-forward neural networks. In Machine Learning and Cybernetics, 2005. Proceedings of 2005 International Conference on 2005 Aug 18 (Vol. 5, pp. 2890-2894). IEEE.

[34] Azad MA, Rocha AM, Fernandes EM. Improved binary artificial fish swarm algorithm for the 0-1 multidimensional knapsack problems. Swarm and Evolutionary Computation. 2014;14:66-75.

[35] Sathya J, Geetha K. Automtic segmentation of lesion from breast DCE-MR image using artificial fish swarm optimization algorithm. Pol J Med Phys Eng. 2017;23(2):29-36.

[36] Yousef WA, Wagner RF, Loew MH. Assessing classifiers from two independent data sets using ROC analysis: a nonparametric approach. IEEE Trans Pattern Anal Mach Intell. 2006;28(11):1809-1817. 